\title{
Le concept de qualité - un défi et une chance
}

\author{
Johannes Brühwiler ${ }^{a}$, Jean-Michel Gaspoz ${ }^{b}$, François Héritierc, Marc Müller ${ }^{\text {, }}$, Lukas Zempe \\ a Responsable du groupe de travail de la qualité de la SSMIG; ' Président du Directoire de la SSMI; ${ }^{c}$ Président SSMG; ${ }^{d}$ Président MFE;
}

e Secrétaire général/Directeur du bureau administratif de la SSMI

Dans le cadre des travaux menés pour la création d'une association commune, la Société Suisse de Médecine Interne Générale SSMI et la Société Suisse de Médecine Générale SSMG ont également décidé de développer un concept de qualité pour la nouvelle société de discipline médicale SSMIG. La qualité constitue en effet un élément capital de cette nouvelle société. Après le vaste rejet auquel s'est heurtée l'idée d'un institut fédéral de la qualité en médecine, il va falloir faire preuve d'initiative.

Un petit groupe de travail a été formé* et a développé, en très peu de temps, un concept qui est présenté ci-dessous. Ce concept fait actuellement l'objet d'une consultation auprès des sociétés participantes.

Le défi qui se pose réside dans le fait que ce concept doit réunir les éléments essentiels d'un développement de la qualité, montrer clairement son utilité pour les patients et les médecins et proposer un élément mesurable satisfaisant aux dispositions de la LAMal.

Dans le domaine de la Médecine Interne Générale (MIG), les patients souffrant de maladies multiples occupent une place importante. Il s'agit donc de mettre en œuvre les divers points de vue, axés sur les maladies et sur les organes, dans un concept de prise en charge efficace orienté sur les patients, et d'en mesurer les résultats.

\section{Etat des lieux}

Dans un premier temps, un regroupement des projets en cours jouant un rôle dans le contexte mentionné a été effectué. Tous contiennent des éléments d'assurance qualité et de développement. De nombreux projets sont en cours avec beaucoup d'engagement et d'excellentes informations techniques. Ils se distinguent tous par une forte orientation pratique, assortie d'une utilité concrète. Depuis les années 1990, les hôpitaux tiennent une liste des complications, laquelle s'est avérée un instrument extrêmement précieux pour la réduction des risques [1]. Des cercles de qualité sont également établis dans le domaine ambulatoire depuis les années 1990. Dès 2014, la SSMI a lancé la campagne «Smarter Medicine» qui se concentre sur les examens et les traitements pouvant être évités, et promeut une médecine basée sur les faits [2]. Les lignes directrices constituent un thème important: elles doivent résumer les conclusions de la médecine basée sur les faits pour qu'elles soient disponibles dans le cadre de la pratique. Souvent fondées sur des travaux internationaux, elles existent dans de nombreux domaines. Leur adéquation à la pratique varie, elles sont souvent bien trop étendues pour le quotidien.

\section{Concept}

Toutes ces informations devraient aider le patient à prendre une décision éclairée, afin qu'une prise en charge adaptée à ses besoins propres puisse être mise en place. Le résultat doit pouvoir être vérifié. Idéalement, il faudrait en déduire un indicateur de qualité. Le concept de qualité de la Médecine Interne Générale (MIG) rassemble désormais ces éléments au sein d'un processus pertinent.

\section{Directives}

Les directives constituent le fondement du travail de qualité en ce sens qu'elles résument les connaissances

La vision de la qualité dans la Médecine Interne Générale

- La fonction d'intégration de la Médecine Interne Générale est renforcée par la collaboration avec toutes les spécialités sur les thèmes relatifs à la qualité.

L'approche généraliste et intégrante de la Médecine Interne Générale fait contrepoids à la fragmentation croissante de la médecine.

Le travail sur la qualité est fondamental pour la reconnaissance de l'importance de la Médecine Interne Générale. 
actuellement disponibles et permettent d'étayer les décisions à prendre. Ces directives doivent être basées sur les faits et préparer les informations de manière à ce qu'elles puissent être facilement accessibles au quotidien. Dans l'idéal, les éléments clés seront rendus disponibles dans un document électronique et seront immédiatement intégrés dans la planification des prises en charge. Ils devront régulièrement être mis à jour afin de refléter l'état actuel des connaissances. Il faut impérativement procéder à une mise en réseau internationale avec des initiatives similaires. La base de connaissances nécessaire à ces directives est identique à celle requise pour la campagne «Smarter Medicine». Les travaux se complètent donc idéalement.

\section{Application des directives}

Les lignes directrices font référence aux maladies. Or, en pratique, ce n'est pas une maladie que nous avons en face de nous, mais un patient avec une personnalité qui lui est propre, une histoire et un environnement. En définitive, une prise en charge est un contrat, passé avec le patient, qui prend en compte ses préférences individuelles. Les directives constituent la base des connaissances sur lesquelles il convient de s'appuyer pour se faire un avis et en définitive prendre une décision.

\section{Shared Decision Making et «Smarter Medicine»}

Les informations doivent être transmises de manière claire et compréhensible dans le cadre d'un processus structuré. Ce processus, appelé Shared Decision Making ou prise de décision partagée, constitue un élément fondamental dans le concept de qualité [3]. Afin de proposer une présentation simple et compréhensible des bénéfices et des risques telle que les médecins et les patients en ont besoin, il convient de rechercher la collaboration avec des institutions établies.

Pour la Shared Decision Making, il est possible de se référer à des travaux essentiels déjà existants [4]. A cet égard, une présentation, simple et basée sur les faits, des bénéfices et des risques de la prise en charge représente, une fois encore, une base indispensable. Les médecins sont formés à l'interprétation et à la transmission d'informations statistiques ainsi qu'à la communication des bénéfices et des risques. Dans le cas de patients souffrant de plusieurs maladies, ce processus est particulièrement important car il est possible que différentes lignes directrices viennent s'appliquer et qu'il faille renoncer à certaines mesures.

Le concept de "Smarter Medicine» repose sur les mêmes fondements que celui de la Shared Decision Making et définit les mesures auxquelles il serait rai-
Concept de qualité de la Médecine Interne Générale

\author{
Directives \\ $\downarrow$ \\ Shared Decision Making - \\ «Smarter Medicine» \\ $\downarrow$ \\ Formulation des objectifs \\ $\downarrow$ \\ Réalisation des objectifs
}

sonnable de renoncer. Ces deux éléments se complètent idéalement et peuvent être réalisés en étroite coordination.

Objectif de prise en charge et réalisation des objectifs: La décision partagée permet de planifier la prise en charge et de formuler, en la matière, un objectif correspondant aux souhaits et aux ressources du patient. Lorsque l'objectif est clairement établi, il est possible d'en mesurer la réalisation.

Nous avons ainsi un indicateur de qualité qui prend en compte la situation individuelle du patient, sur la base des directives, et se concentre sur l'utilité pour le patient. Cela permet d'éviter le risque d'indicateurs abstraits et inadaptés à la situation.

\section{Mise en œuvre}

La mise en œuvre pratique de ce concept exige une approche pluridisciplinaire, les différentes spécialités devant être intégrées dans l'élaboration des directives. Une standardisation formelle veillera à améliorer la disponibilité de ces directives par le biais d'un document électronique. Cela peut être une tâche pour une institution supérieure comme l'ASQM, en collaboration avec eHealth. Le projet dans son ensemble nécessite des ressources considérables, c'est pourquoi une planification soigneuse et une bonne organisation sont essentielles. En revanche, il n'incombe plus à chaque institution d'élaborer ses propres lignes directrices, ce qui représente aussi un potentiel d'économie. L'organisation du projet répartira les travaux sur plusieurs groupes et aura la responsabilité d'assurer une compilation fluide des résultats.

\section{Références}

1 Stäubli M, Suter J. La liste des complications de la Société Suisse de la médecine interne. Bull Méd Suisses. 2004;85(21):1109-16.

2 Amstad H, Gaspoz JM, Zemp L. Guidelines and Choosing wisely: to do's and not to do's. Bull Méd Suisses. 2015;96(5):130-1.

3 Gerber M, Kraft E, Bosshard C. Décision partagée-Médecin et patient décident ensemble. Bull Méd Suisses. 2014;95(50):1883-92.

4 Meier CA. Dans: Gemeinsam zum Entscheid. Dialog Ethik TIF 120, avril 2015. 